\title{
CONTROLLABILITY AND HYERS-ULAM STABILITY RESULTS OF INITIAL VALUE PROBLEMS FOR FRACTIONAL DIFFERENTIAL EQUATIONS VIA GENERALIZED PROPORTIONAL-CAPUTO FRACTIONAL DERIVATIVE
}

\author{
MOHAMED I. ABBAS
}

Received 06 October, 2020

\begin{abstract}
This paper concerns the investigation of controllability and Hyers-Ulam stability of initial value problems for fractional differential equations via generalized proportional-Caputo fractional derivatives. The main results are obtained by means of the Krasnoselskii's fixed point theorem.
\end{abstract}

2010 Mathematics Subject Classification: 26A33; 93B05; 34K35

Keywords: Hyers-Ulam stability, controllability, proportional-Caputo derivatives, Krasnoselskii's fixed point theorem

\section{INTRODUCTION}

Fractional differential equations have been studied extensively in the literature because of their applications in various fields of engineering and science; see the books $[16,18,22]$. In the few past years, the variety of definitions of fractional operators has become visible to those interested in fractional calculus.Here, we focus on the most ranking kinds including Liouville, Caputo, Hadamard, Atangana-Baleanu, CaputoFabrizio derivatives and etc. For instance, see the books $[15,23]$ and the papers $[3,8,19-21]$ and the references quoted therein.

Recently, Khalil et al. [14] introduce a new definition of fractional derivative, called the conformable fractional derivative, with an obstacle that it does not tend to the original function as the order $\alpha$ tends to zero. The new definition has attracted good efforts of many researchers to establish some useful results; see, for example, $[5-7,17,26]$.

In control theory, a proportional derivative controller for controller output $u$ at time $t$ with two tuning parameters has the algorithm

$$
u(t)=\kappa_{P} \mathcal{E}(t)+\kappa_{d} \frac{d}{d t} \mathcal{E}(t),
$$


where $\kappa_{P}$ and $\kappa_{d}$ are the proportional control parameter and the derivative control parameter, respectively. The function $\mathcal{E}$ is the error between the state variable and the process variable. This control law enables Dawei et al. [11] to present the control of complex networks models.

Inspired by the above concept of the proportional derivative controller, Anderson et al. [2] were able to define the proportional (conformable) derivative of order $\alpha$ by

$$
{ }_{0}^{P} D_{t}^{\alpha} g(t)=k_{1}(\alpha, t) g(t)+k_{0}(\alpha, t) g^{\prime}(t),
$$

where $g$ is differentiable function and $k_{0}, k_{1}:[0,1] \times \mathbb{R} \rightarrow[0, \infty)$ are continuous functions of the variable $t$ and the parameter $\alpha \in[0,1]$ which satisfy the following conditions for all $t \in \mathbb{R}$ :

$$
\begin{aligned}
& \lim _{\alpha \rightarrow 0^{+}} k_{0}(\alpha, t)=0, \quad \lim _{\alpha \rightarrow 1^{-}} k_{0}(\alpha, t)=1, k_{0}(\alpha, t) \neq 0, \alpha \in(0,1], \\
& \lim _{\alpha \rightarrow 0^{+}} k_{1}(\alpha, t)=1, \quad \lim _{\alpha \rightarrow 1^{-}} k_{1}(\alpha, t)=0, k_{1}(\alpha, t) \neq 0, \alpha \in[0,1) .
\end{aligned}
$$

This newly defined local derivative tends to the original function as the order $\alpha$ tends to zero and hence improved the conformable derivatives. In [13], Jarad et al. discussed a special case of the proportional derivatives when $k_{1}(\alpha, t)=1-\alpha$ and $k_{0}(\alpha, t)=\alpha$.

In [4], Baleanu et al. proposed a new more general proportional fractional derivative as a linear combination of a Riemann-Liouville integral and a Caputo derivative, also they obtained some amazing results relevant to the newly hybrid fractional operator such as the Laplace transform and its inversion. Further, they solved some differential equations involved that new hybrid derivative and got the solution in terms of a new bivariate Mittag-Leffler function.

Inspired by the new results in [4], we investigate initial value problems for fractional differential equations via generalized proportional-Caputo fractional derivatives. Precisely, we consider the following IVP:

$$
\left\{\begin{array}{l}
{ }_{0}^{P C} D_{t}^{\alpha} x(t)=f(t, x(t))+B u(t), t \in J=[0, b], b<\infty, \\
x(0)=x_{0} \in \mathbb{R},
\end{array}\right.
$$

where ${ }_{0}^{P C} D_{t}^{\alpha}$ denotes the proportional-Caputo fractional derivative of order $\alpha \in(0,1]$, the function $f: J \times \mathbb{R} \rightarrow \mathbb{R}$ is continuous, the control function $u(\cdot)$ is given in $L^{2}(J, U)$, a Banach space of admissible control functions with $U$ as a Banach space, and $B$ is a bounded linear operator from $U$ to $\mathbb{R}$.

Controllability is one of the fundamental notions of modern control theory, which enables one to steer the control system from an arbitrary initial state to an arbitrary final state using the set of admissible controls where initial and final state may vary over the entire space. The problem of controllability of nonlinear systems represented by fractional differential equations has been extensively studied by several authors; see, for example, $[1,9,10,25]$ and the references therein. 
To our knowledge there are no similar contributions to the controllability and Hyers-Ulam stability of fractional differential equations via generalized proportionalCaputo fractional derivatives.

\section{PReliminaries}

In this section we collect some definitions, properties and propositions of the new generalized proportional-Caputo hybrid fractional derivative.

Definition 1 ([4]). The proportional-Caputo hybrid fractional derivative of order $\alpha \in(0,1)$ of a differentiable function $g(t)$ is given by

$$
{ }_{0}^{P C} D_{t}^{\alpha} g(t)=\frac{1}{\Gamma(1-\alpha)} \int_{0}^{t}\left(k_{1}(\alpha, \tau) g(t)+k_{0}(\alpha, \tau) g^{\prime}(t)\right)(t-\tau)^{-\alpha} d \tau,
$$

where the function space domain is given by requiring that $g$ is differentiable and both $g$ and $g^{\prime}$ are locally $L^{1}$ functions on the positive reals.

Definition 2 ([4]). The inverse operator of the proportional-Caputo hybrid fractional derivative of order $\alpha \in(0,1)$ is given by

$$
{ }_{0}^{P C} I_{t}^{\alpha} g(t)=\int_{0}^{t} \exp \left(-\int_{u}^{t} \frac{k_{1}(\alpha, s)}{k_{0}(\alpha, s)} d s\right) \frac{{ }_{0}^{R L} D_{u}^{1-\alpha} g(u)}{k_{0}(\alpha, u)} d u,
$$

where ${ }_{0}^{R L} D_{u}^{1-\alpha}$ denotes the Riemann-Liouville fractional derivative of order $1-\alpha$ and is given by

$$
{ }_{0}^{R L} D_{u}^{1-\alpha} g(u)=\frac{1}{\Gamma(\alpha)} \frac{d}{d u} \int_{0}^{u}(u-s)^{\alpha-1} g(s) d s .
$$

For more details, we refer the reader to the book of Kilbas et al. [15].

Proposition 1 ([4]). The following inversion relations

$$
\begin{gathered}
{ }_{0}^{P C} D_{t}^{\alpha}{ }_{0}^{P C} I_{t}^{\alpha} g(t)=g(t)-\frac{t^{-\alpha}}{\Gamma(1-\alpha)} \lim _{t \rightarrow 0}{ }_{0}^{R L} I_{t}^{\alpha} g(t), \\
{ }_{0}^{P C} I_{t}^{\alpha}{ }_{0}^{P C} D_{t}^{\alpha} g(t)=g(t)-\exp \left(-\int_{0}^{t} \frac{k_{1}(\alpha, s)}{k_{0}(\alpha, s)} d s\right) g(0)
\end{gathered}
$$

are satisfied.

Proposition 2 ([4]). The proportional-Caputo hybrid fractional derivative operator ${ }_{0}^{P C} D_{t}^{\alpha}$ is non-local and singular.

Remark 1 ([4]). In the limiting cases $\alpha \rightarrow 0$ and $\alpha \rightarrow 1$, we recover the following special cases:

$$
\begin{aligned}
& \lim _{\alpha \rightarrow 0}{ }_{0}^{P C} D_{t}^{\alpha} g(t)=\int_{0}^{t} g(\tau) d \tau, \\
& \lim _{\alpha \rightarrow 1}{ }_{0}^{P C} D_{t}^{\alpha} g(t)=g(t) .
\end{aligned}
$$


Theorem 1 (Krasnoselskii's fixed point theorem, [24]). Let $\Omega$ be a closed convex and non-empty subset of a Banach space $\mathbb{X}$. Let $\mathcal{P}_{1}$ and $\mathcal{P}_{2}$, be two operators such that

(i) $\mathcal{P}_{1} x+\mathcal{P}_{2} y \in \Omega$, for all $x, y \in \Omega$,

(ii) $\mathcal{P}_{1}$ is compact and continuous,

(iii) $\mathcal{P}_{2}$ is a contraction mapping.

Then there exists $z \in \Omega$ such that $z=\mathscr{P}_{1} z+\mathcal{P}_{2} z$.

\section{Controllability Results}

In this section, we employ the generalized proportional Caputo fractional derivative operator to discuss the controllability of the IVP (1.3).

Let $C(J, \mathbb{R})$ be the Banach space of all real-valued continuous functions from $J$ into $\mathbb{R}$ equipped by the norm $\|x\|=\sup _{t \in[0, T]}|x(t)|$.

Firstly, we consider the following auxiliary lemma.

Lemma 1. Let $0<\alpha \leq 1$ and $h \in C(J, \mathbb{R})$. Then the solution of the following linear fractional differential equation

$$
\left\{\begin{array}{l}
{ }_{0}^{P C} D_{t}^{\alpha} x(t)=h(t), t \in J, \\
x(0)=x_{0},
\end{array}\right.
$$

is equivalent to the Volterra integral equation

$$
\begin{aligned}
x(t)= & \exp \left(-\int_{0}^{t} \frac{k_{1}(\alpha, s)}{k_{0}(\alpha, s)} d s\right) x_{0} \\
& +\frac{1}{\Gamma(\alpha-1)} \int_{0}^{t} \int_{0}^{u} \exp \left(-\int_{u}^{t} \frac{k_{1}(\alpha, s)}{k_{0}(\alpha, s)} d s\right) \frac{(u-\tau)^{\alpha-2}}{k_{0}(\alpha, u)} h(\tau) d \tau d u .
\end{aligned}
$$

Proof. Applying the operator ${ }_{0}^{P C} I_{t}^{\alpha}(\cdot)$ on both sides of (3.1), we get

$$
{ }_{0}^{P C} I_{t}^{\alpha} \quad{ }_{0}^{P C} D_{t}^{\alpha} x(t)={ }_{0}^{P C} I_{t}^{\alpha} h(t) .
$$

Using (2.2) and (2.3) together with Proposition 1, we get

$$
x(t)-\exp \left(-\int_{0}^{t} \frac{k_{1}(\alpha, s)}{k_{0}(\alpha, s)} d s\right) x(0)=\int_{0}^{t} \exp \left(-\int_{u}^{t} \frac{k_{1}(\alpha, s)}{k_{0}(\alpha, s)} d s\right) \frac{{ }_{0}^{R L} D_{u}^{1-\alpha} h(u)}{k_{0}(\alpha, u)} d u .
$$

In view of the following elementary fact:

$$
{ }_{0}^{R L} D_{u}^{1-\alpha} h(u)={ }_{0}^{R L} I_{u}^{-(1-\alpha)} h(u)={ }_{0}^{R L} I_{u}^{\alpha-1} h(u)=\frac{1}{\Gamma(\alpha-1)} \int_{0}^{u}(u-\tau)^{\alpha-2} h(\tau) d \tau,
$$

one can easily obtain the desired integral equation (3.3). The converse follows by direct computation. This completes the proof. 
By virtue of Lemma 1, the solution of the IVP (1.3) is given by

$$
\begin{aligned}
x(t)= & \exp \left(-\int_{0}^{t} \frac{k_{1}(\alpha, s)}{k_{0}(\alpha, s)} d s\right) x_{0} \\
& +\frac{1}{\Gamma(\alpha-1)} \int_{0}^{t} \int_{0}^{u} \exp \left(-\int_{u}^{t} \frac{k_{1}(\alpha, s)}{k_{0}(\alpha, s)} d s\right) \frac{(u-\tau)^{\alpha-2}}{k_{0}(\alpha, u)} f(\tau, x(\tau)) d \tau d u \\
& +\frac{1}{\Gamma(\alpha-1)} \int_{0}^{t} \int_{0}^{u} \exp \left(-\int_{u}^{t} \frac{k_{1}(\alpha, s)}{k_{0}(\alpha, s)} d s\right) \frac{(u-\tau)^{\alpha-2}}{k_{0}(\alpha, u)} B u_{x}(\tau) d \tau d u .
\end{aligned}
$$

The following definition is helpful in the discussion of the controllability of the IVP (1.3).

Definition 3. The IVP (1.3) is said to be controllable on the interval $J$ if, for every $x_{0}, x_{1} \in \mathbb{R}$, there exists a control $u \in L^{2}(J, U)$ such that a solution $x$ of equation (1.3) satisfies $x(b)=x_{1}$.

The following assumptions will be imposed.

(A1) The function $f: J \times \mathbb{R} \rightarrow \mathbb{R}$ is continuous.

(A2) There exists a constant $L>0$ such that

$$
|f(t, x)-f(t, y)| \leq L|x-y|, \text { for all } t \in J, x, y \in \mathbb{R} .
$$

(A3) The linear operator $W: L^{2}(J, U) \rightarrow \mathbb{R}$, defined by

$$
W u=\frac{1}{\Gamma(\alpha-1)} \int_{0}^{b} \int_{0}^{u} \exp \left(-\int_{u}^{b} \frac{k_{1}(\alpha, s)}{k_{0}(\alpha, s)} d s\right) \frac{(u-\tau)^{\alpha-2}}{k_{0}(\alpha, u)} B u(\tau) d \tau d u
$$

has an induced inverse operator $W^{-1}$ which takes values in $L^{2}(J, U) / \operatorname{ker} W$, where the kernel space of $W$ is defined by ker $W=\left\{x \in L^{2}(J, U): W x=0\right\}$ and there exist constants $M_{1}, M_{2}>0$ such that $\|B\| \leq M_{1}$ and $\left\|W^{-1}\right\| \leq M_{2}$.

Now we formulate the first main theorem of the paper.

Theorem 2. If the assumptions $(\mathbf{A 1})-(\mathbf{A 3})$ are satisfied. Then the IVP (1.3) is controllable on J, provided that

$$
\frac{M_{1} M_{2} b^{2 \alpha} L}{M_{k}^{2} \Gamma^{2}(\alpha+1)}<1,
$$

where $\inf _{t \in J}\left|k_{0}(\alpha, t)\right|=M_{k} \neq 0$.

Proof. Set $\sup _{t \in J}|f(t, 0)|=M_{f}<\infty$.

We consider the set $\left.\mathcal{B}_{r}=\{x \in C(J, \mathbb{R}):\|x\| \leq r)\right\}$ with $r \geq \frac{\Lambda_{1}}{1-\Lambda_{2}}$.

For the purpose of expediency, we define the two constants $\Lambda_{1}>0$ and $0<\Lambda_{2}<1$ as

$$
\Lambda_{1}=\left|x_{0}\right|+\frac{M_{f} b^{\alpha}}{M_{k} \Gamma(\alpha+1)}+\frac{M_{1} M_{2} b^{\alpha}}{M_{k} \Gamma(\alpha+1)}\left[\left|x_{1}\right|+\left|x_{0}\right|+\frac{M_{f} b^{\alpha}}{M_{k} \Gamma(\alpha+1)}\right]
$$




$$
\Lambda_{2}=\frac{L b^{\alpha}}{M_{k} \Gamma(\alpha+1)}\left(1+\frac{M_{1} M_{2} b^{\alpha}}{M_{k} \Gamma(\alpha+1)}\right) .
$$

Define the control $u_{x}(t)$ by

$$
\begin{aligned}
u_{x}(t)= & W^{-1}\left[x_{1}-\exp \left(-\int_{0}^{b} \frac{k_{1}(\alpha, s)}{k_{0}(\alpha, s)} d s\right) x_{0}\right. \\
& \left.+\frac{1}{\Gamma(\alpha-1)} \int_{0}^{b} \int_{0}^{u} \exp \left(-\int_{u}^{b} \frac{k_{1}(\alpha, s)}{k_{0}(\alpha, s)} d s\right) \frac{(u-\tau)^{\alpha-2}}{k_{0}(\alpha, u)} f(\tau, x(\tau)) d \tau d u\right](t), t \in J .
\end{aligned}
$$

Later, we shall use the following estimates:

$$
\begin{aligned}
& \left\|u_{x}\right\|=\sup _{t \in J}\left|u_{x}(t)\right| \\
& \leq M_{2} \sup _{t \in J}\left\{\left|x_{1}\right|+\left|x_{0}\right|+\frac{1}{\Gamma(\alpha-1)} \int_{0}^{b} \int_{0}^{u} \frac{(u-\tau)^{\alpha-2}}{\left|k_{0}(\alpha, u)\right|}|(f(\tau, x(\tau))-f(\tau, 0))+f(\tau, 0)| d \tau d u\right\} \\
& \leq M_{2} \sup _{t \in J}\left\{\left|x_{1}\right|+\left|x_{0}\right|+\frac{1}{\Gamma(\alpha-1)} \int_{0}^{b} \int_{0}^{u} \frac{(u-\tau)^{\alpha-2}}{\left|k_{0}(\alpha, u)\right|}(|f(\tau, x(\tau))-f(\tau, 0)|+|f(\tau, 0)|) d \tau d u\right\} \\
& \leq M_{2} \sup _{t \in J}\left\{\left|x_{1}\right|+\left|x_{0}\right|+\frac{1}{\Gamma(\alpha-1)} \int_{0}^{b} \int_{0}^{u} \frac{(u-\tau)^{\alpha-2}}{\left|k_{0}(\alpha, u)\right|}(L|x(\tau)|+|f(\tau, 0)|) d \tau d u\right\} \\
& \leq M_{2}\left[\left|x_{1}\right|+\left|x_{0}\right|+\frac{b^{\alpha}}{M_{k} \Gamma(\alpha+1)}\left(L\|x\|+M_{f}\right)\right]
\end{aligned}
$$

and

$$
\begin{aligned}
\left\|u_{x}-u_{y}\right\| & =\sup _{t \in J}\left|u_{x}(t)-u_{y}(t)\right| \\
& \leq M_{2} \sup _{t \in J}\left\{\frac{1}{\Gamma(\alpha-1)} \int_{0}^{b} \int_{0}^{u} \frac{(u-\tau)^{\alpha-2}}{\left|k_{0}(\alpha, u)\right|}|f(\tau, x(\tau))-f(\tau, y(\tau))| d \tau d u\right\} \\
& \leq \frac{M_{2} L}{M_{k} \Gamma(\alpha-1)} \sup _{t \in J}\left\{\int_{0}^{b} \int_{0}^{u}(u-\tau)^{\alpha-2}|x(\tau)-y(\tau)| d \tau d u\right\} \\
& \leq \frac{M_{2} L b^{\alpha}}{M_{k} \Gamma(\alpha+1)}\|x-y\| .
\end{aligned}
$$

Using the control $u_{x}(t)$, we define the operators $\mathcal{P}_{1}, \mathcal{P}_{2}$ on $\mathcal{B}_{r}$ as:

$$
\begin{aligned}
\left(\mathcal{P}_{1} x\right)(t)= & \exp \left(-\int_{0}^{t} \frac{k_{1}(\alpha, s)}{k_{0}(\alpha, s)} d s\right) x_{0} \\
& +\frac{1}{\Gamma(\alpha-1)} \int_{0}^{t} \int_{0}^{u} \exp \left(-\int_{u}^{t} \frac{k_{1}(\alpha, s)}{k_{0}(\alpha, s)} d s\right) \frac{(u-\tau)^{\alpha-2}}{k_{0}(\alpha, u)} f(\tau, x(\tau)) d \tau d u \\
\left(\mathcal{P}_{2} x\right)(t)= & \frac{1}{\Gamma(\alpha-1)} \int_{0}^{t} \int_{0}^{u} \exp \left(-\int_{u}^{t} \frac{k_{1}(\alpha, s)}{k_{0}(\alpha, s)} d s\right) \frac{(u-\tau)^{\alpha-2}}{k_{0}(\alpha, u)} B u_{x}(\tau) d \tau d u
\end{aligned}
$$


Clearly, one can notice that $\left(\mathcal{P}_{1} x+\mathcal{P}_{2} x\right)(b)=x_{1}$. This means that $u_{x}$ steers the IVP (1.3) from $x_{0}$ to $x_{1}$ in finite time $b$, which implies that the IVP (1.3) is controllable on $J$.

The proof is divided into three main steps.

Step 1. $\mathcal{P}_{1} x+\mathcal{P}_{2} y \in \mathcal{B}_{r}, \quad \forall x, y \in \mathcal{B}_{r}$

For each $t \in J$ and $x, y \in \mathcal{B}_{r}$, using (3.5), one has

$$
\begin{aligned}
\left\|\mathcal{P}_{1} x+\mathcal{P}_{2} y\right\|= & \sup _{t \in J}\left|\left(\mathcal{P}_{1} x\right)(t)+\left(\mathcal{P}_{2} y\right)(t)\right| \\
\leq & \sup _{t \in J}\left\{\exp \left(-\int_{0}^{t} \frac{k_{1}(\alpha, s)}{k_{0}(\alpha, s)} d s\right)\left|x_{0}\right|\right. \\
& +\frac{1}{\Gamma(\alpha-1)} \int_{0}^{b} \int_{0}^{u} \exp \left(-\int_{u}^{t} \frac{k_{1}(\alpha, s)}{k_{0}(\alpha, s)} d s\right) \\
& \times \frac{(u-\tau)^{\alpha-2}}{\left|k_{0}(\alpha, u)\right|}|(f(\tau, x(\tau))-f(\tau, 0))+f(\tau, 0)| d \tau d u \\
& \left.+\frac{1}{\Gamma(\alpha-1)} \int_{0}^{b} \int_{0}^{u} \exp \left(-\int_{u}^{t} \frac{k_{1}(\alpha, s)}{k_{0}(\alpha, s)} d s\right) \frac{(u-\tau)^{\alpha-2}}{\left|k_{0}(\alpha, u)\right|}\left|B u_{y}(\tau)\right| d \tau d u\right\} \\
\leq & \left|x_{0}\right|+\frac{1}{M_{k} \Gamma(\alpha-1)} \int_{0}^{b} \int_{0}^{u}(u-\tau)^{\alpha-2}\left(L\|x\|+M_{f}\right) d \tau d u \\
& +\frac{1}{M_{k} \Gamma(\alpha-1)} \int_{0}^{b} \int_{0}^{u}(u-\tau)^{\alpha-2}\|B\| \|\left|u_{y}\right| \mid d \tau d u \\
\leq & \left|x_{0}\right|+\frac{b^{\alpha}}{M_{k} \Gamma(\alpha+1)}\left(L r+M_{f}\right) \\
& +\frac{M_{1} M_{2} b^{\alpha}}{M_{k} \Gamma(\alpha+1)}\left[\left|x_{1}\right|+\left|x_{0}\right|+\frac{b^{\alpha}}{M_{k} \Gamma(\alpha+1)}\left(L r+M_{f}\right)\right] \\
\leq & \left|x_{0}\right|+\frac{M_{f} b^{\alpha}}{M_{k} \Gamma(\alpha+1)}+\frac{M_{1} M_{2} b^{\alpha}}{M_{k} \Gamma(\alpha+1)}\left[\left|x_{1}\right|+\left|x_{0}\right|+\frac{M_{f} b^{\alpha}}{M_{k} \Gamma(\alpha+1)}\right] \\
& +\frac{L b^{\alpha}}{M_{k} \Gamma(\alpha+1)}\left(1+\frac{M_{1} M_{2} b^{\alpha}}{M_{k} \Gamma(\alpha+1)}\right) r \\
= & \Lambda_{1}+\Lambda_{2} r \leq r .
\end{aligned}
$$

Thus, we conclude that $\mathcal{P}_{1} x+\mathcal{P}_{2} y \in \mathcal{B}_{r}$.

Step 2. $\mathcal{P}_{1}$ is compact and continuous. Firstly, we show that $\mathscr{P}_{1}$ is continuous. Let $\left\{x_{n}\right\}$ be a sequence such that $x_{n} \rightarrow x$ as $n \rightarrow \infty$ in $\mathcal{B}_{r}$. Thus, for each $t \in J$, we have

$$
\begin{aligned}
\left\|\mathcal{P}_{1} x_{n}-\mathcal{P}_{1} x\right\| & =\sup _{t \in J}\left|\left(\mathcal{P}_{1} x_{n}\right)(t)-\left(\mathcal{P}_{1} x\right)(t)\right| \\
& \leq \frac{1}{M_{k} \Gamma(\alpha-1)} \int_{0}^{t} \int_{0}^{u}(u-\tau)^{\alpha-2} \|\left(f\left(\cdot, x_{n}(\cdot)\right)-f(\cdot, x(\cdot)) \| d \tau d u .\right.
\end{aligned}
$$


Therefore, the continuity of $f$ implies that $\mathcal{P}_{1}$ is continuous.

Next, we show that $\mathcal{P}_{1}$ is uniformly bounded on $\mathcal{B}_{r}$. For each $t \in J$ and $x \in \mathcal{B}_{r}$, one has

$$
\begin{aligned}
\left\|\mathcal{P}_{1} x\right\|= & \sup _{t \in J}\left|\left(\mathcal{P}_{1} x\right)(t)\right| \\
\leq & \sup _{t \in J}\left\{\exp \left(-\int_{0}^{t} \frac{k_{1}(\alpha, s)}{k_{0}(\alpha, s)} d s\right)\left|x_{0}\right|+\frac{1}{\Gamma(\alpha-1)} \int_{0}^{b} \int_{0}^{u} \exp \left(-\int_{u}^{t} \frac{k_{1}(\alpha, s)}{k_{0}(\alpha, s)} d s\right)\right. \\
& \left.\times \frac{(u-\tau)^{\alpha-2}}{\left|k_{0}(\alpha, u)\right|}|(f(\tau, x(\tau))-f(\tau, 0))+f(\tau, 0)| d \tau d u\right\} \\
\leq & \left|x_{0}\right|+\frac{b^{\alpha}}{M_{k} \Gamma(\alpha+1)}\left(L r+M_{f}\right),
\end{aligned}
$$

which implies that $\mathcal{P}_{1}$ is uniformly bounded on $\mathcal{B}_{r}$.

It remains to show that $\mathcal{P}_{1}$ is equicontinuous. For each $t_{1}, t_{2} \in J, t_{1}<t_{2}$ and $x \in \mathcal{B}_{r}$, one obtain that:

$$
\begin{aligned}
\| & \left(\mathcal{P}_{1} x\right)\left(t_{2}\right)-\left(\mathcal{P}_{1} x\right)\left(t_{1}\right) \| \\
\leq & \left\|\exp \left(-\int_{0}^{t_{2}} \frac{k_{1}(\alpha, s)}{k_{0}(\alpha, s)} d s\right) x_{0}-\exp \left(-\int_{0}^{t_{1}} \frac{k_{1}(\alpha, s)}{k_{0}(\alpha, s)} d s\right) x_{0}\right\| \\
& +\frac{1}{\Gamma(\alpha-1)} \| \int_{0}^{t_{2}} \int_{0}^{u}\left[\exp \left(-\int_{0}^{t_{2}} \frac{k_{1}(\alpha, s)}{k_{0}(\alpha, s)} d s\right)-\exp \left(-\int_{0}^{t_{1}} \frac{k_{1}(\alpha, s)}{k_{0}(\alpha, s)} d s\right)\right] \\
& \times \frac{(u-\tau)^{\alpha-2}}{k_{0}(\alpha, u)}(f(\tau, x(\tau)) d \tau d u \| \\
& +\frac{1}{\Gamma(\alpha-1)} \| \int_{t_{1}}^{t_{2}} \int_{0}^{u} \exp \left(-\int_{0}^{t_{1}} \frac{k_{1}(\alpha, s)}{k_{0}(\alpha, s)} d s\right) \frac{(u-\tau)^{\alpha-2}}{k_{0}(\alpha, u)}(f(\tau, x(\tau)) d \tau d u \| \\
= & \left\|\frac{k_{1}(\alpha, \xi)}{k_{0}(\alpha, \xi)} x_{0} \exp \left(-\int_{0}^{\xi} \frac{k_{1}(\alpha, s)}{k_{0}(\alpha, s)} d s\right)\left(t_{2}-t_{1}\right)\right\| \\
& +\frac{1}{\Gamma(\alpha-1)} \| \int_{0}^{t_{2}} \int_{0}^{u} \frac{k_{1}(\alpha, \xi)}{k_{0}(\alpha, \xi)} \exp \left(-\int_{0}^{\xi} \frac{k_{1}(\alpha, s)}{k_{0}(\alpha, s)} d s\right) \\
& \left(t_{2}-t_{1}\right) \frac{(u-\tau)^{\alpha-2}}{k_{0}(\alpha, u)}(f(\tau, x(\tau)) d \tau d u \| \\
& +\frac{1}{\Gamma(\alpha-1)} \| \int_{t_{1}}^{t_{2}} \int_{0}^{u} \exp \left(-\int_{0}^{t_{1}} \frac{k_{1}(\alpha, s)}{k_{0}(\alpha, s)} d s\right) \frac{(u-\tau)^{\alpha-2}}{k_{0}(\alpha, u)}(f(\tau, x(\tau)) d \tau d u \| \\
\leq & \left|\frac{k_{1}(\alpha, \xi)}{k_{0}(\alpha, \xi)} x_{0}\right|\left(t_{2}-t_{1}\right)+\frac{\bar{f}}{\Gamma(\alpha+1)}\left|\frac{k_{1}(\alpha, \xi)}{k_{0}^{2}(\alpha, \xi)}\right| t_{2}\left(t_{2}-t_{1}\right)+\frac{\bar{f}}{\Gamma(\alpha+1)}\left|\frac{1}{k_{0}(\alpha, \xi)}\right|\left(t_{2}^{\alpha}-t_{1}^{\alpha}\right),
\end{aligned}
$$


where $\bar{f}=\sup _{t \in J \times \mathcal{B}_{r}} \mid f\left(t, x(t) \mid\right.$ and $\xi \in\left(t_{1}, t_{2}\right)$. As $t_{1} \rightarrow t_{2}$, the right hand side of the above inequality tends to zero independently of $x \in \mathcal{B}_{r}$. As a consequence of the Arzelà-Ascoli theorem, we deduce that $\mathcal{P}_{1}$ is compact on $\mathcal{B}_{r}$.

Step 3. $\mathcal{P}_{2}$ is a contraction on $\mathcal{B}_{r}$.

For each $t \in J$ and $x, y \in \mathcal{B}_{r}$, using (3.6), one has

$$
\begin{aligned}
\| \mathcal{P}_{2} x & -\mathcal{P}_{2} y \|=\sup _{t \in J}\left|\left(\mathcal{P}_{2} x\right)(t)-\left(\mathcal{P}_{2} y\right)(t)\right| \\
& =\sup _{t \in J}\left\{\frac{1}{\Gamma(\alpha-1)} \int_{0}^{t} \int_{0}^{u} \exp \left(-\int_{u}^{t} \frac{k_{1}(\alpha, s)}{k_{0}(\alpha, s)} d s\right) \frac{(u-\tau)^{\alpha-2}}{k_{0}(\alpha, u)} B\left(u_{x}(\tau)-u_{y}(\tau)\right) d \tau d u\right\} \\
& \leq \frac{M_{1} b^{\alpha}}{M_{k} \Gamma(\alpha+1)}\left\|u_{x}-u_{y}\right\| \\
& \leq \frac{M_{1} M_{2} b^{2 \alpha} L}{M_{k}^{2} \Gamma^{2}(\alpha+1)}\|x-y\| .
\end{aligned}
$$

In view of the condition (3.4), we conclude that $\mathcal{P}_{2}$ is a contraction mapping.

Therefore, all the assumptions of Krasnoselskii's fixed point theorem (Theorem 1) are satisfied. Hence, the IVP (1.3) is controllable on $J$. This completes the proof.

\section{Hyers-Ulam Stability}

Here, we elucidate Hyers-Ulam stability of the IVP (1.3). We begin with the following essential definition.

Definition 4 ([12]). The integral equation (3.3) is said to be Hyers-Ulam stable, if there exists a constant $\mu>0$ satisfying: for every $\varepsilon>0$, if

$$
\begin{aligned}
& \mid x(t)-\exp \left(-\int_{0}^{t} \frac{k_{1}(\alpha, s)}{k_{0}(\alpha, s)} d s\right) x_{0} \\
& -\frac{1}{\Gamma(\alpha-1)} \int_{0}^{t} \int_{0}^{u} \exp \left(-\int_{u}^{t} \frac{k_{1}(\alpha, s)}{k_{0}(\alpha, s)} d s\right) \frac{(u-\tau)^{\alpha-2}}{k_{0}(\alpha, u)} f(\tau, x(\tau)) d \tau d u \\
& -\frac{1}{\Gamma(\alpha-1)} \int_{0}^{t} \int_{0}^{u} \exp \left(-\int_{u}^{t} \frac{k_{1}(\alpha, s)}{k_{0}(\alpha, s)} d s\right) \frac{(u-\tau)^{\alpha-2}}{k_{0}(\alpha, u)} B u_{x}(\tau) d \tau d u \mid \leq \varepsilon
\end{aligned}
$$

there exists a continuous function $x^{*}(t)$ satisfying

$$
\begin{aligned}
x^{*}(t) & =\exp \left(-\int_{0}^{t} \frac{k_{1}(\alpha, s)}{k_{0}(\alpha, s)} d s\right) x_{0} \\
& +\frac{1}{\Gamma(\alpha-1)} \int_{0}^{t} \int_{0}^{u} \exp \left(-\int_{u}^{t} \frac{k_{1}(\alpha, s)}{k_{0}(\alpha, s)} d s\right) \frac{(u-\tau)^{\alpha-2}}{k_{0}(\alpha, u)} f\left(\tau, x^{*}(\tau)\right) d \tau d u \\
& +\frac{1}{\Gamma(\alpha-1)} \int_{0}^{t} \int_{0}^{u} \exp \left(-\int_{u}^{t} \frac{k_{1}(\alpha, s)}{k_{0}(\alpha, s)} d s\right) \frac{(u-\tau)^{\alpha-2}}{k_{0}(\alpha, u)} B u_{x^{*}}(\tau) d \tau d u,
\end{aligned}
$$


such that

$$
\left|x(t)-x^{*}(t)\right| \leq \mu \varepsilon, \quad \forall t \in J .
$$

Theorem 3. Assume that the assumptions $(\mathbf{A 1})-(\mathbf{A 3})$ are satisfied. Then the IVP (1.3) is Hyers-Ulam stable.

Proof. With the help of Theorem 2, let $x(t)$ be unique solution of (3.3) and $x^{*}(t)$ be any other solution satisfying (4.1). Then, by a similar way in the proof of Theorem 2 and by virtue of (3.6), one has

$$
\begin{aligned}
\left|x(t)-x^{*}(t)\right| \leq & \frac{1}{M_{k} \Gamma(\alpha-1)} \int_{0}^{t} \int_{0}^{u}(u-\tau)^{\alpha-2}\left|f(\tau, x(\tau))-f\left(\tau, x^{*}(\tau)\right)\right| d \tau d u \\
& +\frac{M_{1}}{M_{k} \Gamma(\alpha-1)} \int_{0}^{t} \int_{0}^{u}(u-\tau)^{\alpha-2}\left|u_{x}(\tau)-u_{x^{*}}(\tau)\right| d \tau d u \\
\leq & \frac{L}{M_{k} \Gamma(\alpha-1)} \int_{0}^{t} \int_{0}^{u}(u-\tau)^{\alpha-2}\left|x(\tau)-x^{*}(\tau)\right| d \tau d u \\
& +\frac{M_{1}}{M_{k} \Gamma(\alpha-1)} \int_{0}^{t} \int_{0}^{u}(u-\tau)^{\alpha-2}\left|u_{x}(\tau)-u_{x^{*}}(\tau)\right| d \tau d u \\
\leq & \left(\frac{b^{\alpha} L}{M_{k} \Gamma(\alpha+1)}+\frac{M_{1} M_{2} b^{2 \alpha} L}{M_{k}^{2} \Gamma^{2}(\alpha+1)}\right)\left\|x-x^{*}\right\| \\
= & \mu\left\|x-x^{*}\right\|,
\end{aligned}
$$

where

$$
\mu:=\frac{b^{\alpha} L}{M_{k} \Gamma(\alpha+1)}+\frac{M_{1} M_{2} b^{2 \alpha} L}{M_{k}^{2} \Gamma^{2}(\alpha+1)} .
$$

Therefore, the integral equation (3.3) is Hyers-Ulam stable. Consequently, the IVP (1.3) is Hyers-Ulam stable. The proof is finished.

\section{REFERENCES}

[1] H. M. Ahmed, M. M. El-Borai, A. O. El Bab, and M. E. Ramadan, "Controllability and constrained controllability for nonlocal Hilfer fractional differential systems with Clarke's subdifferential," Journal of Inequalities and Applications, vol. 2019, no. 1, pp. 1-23, 2019, doi: 10.1186/s13660019-2184-6.

[2] D. R. Anderson and D. J. Ulness, "Newly defined conformable derivatives," Adv. Dyn. Syst. Appl, vol. 10, no. 2, pp. 109-137, 2015, doi: 10.13140/RG.2.1.1744.9444.

[3] A. Atangana and D. Baleanu, "New fractional derivatives with nonlocal and non-singular kernel: Theory and application to heat transfer model," Thermal Science, vol. 20, no. 2, pp. 763-769, 2016, doi: 10.2298/TSCI160111018A

[4] D. Baleanu, A. Fernandez, and A. Akgül, "On a fractional operator combining proportional and classical differintegrals," Mathematics, vol. 8, no. 3, p. 360, 2020, doi: 10.3390/math8030360.

[5] H. Batarfi, J. Losada, J. J. Nieto, and W. Shammakh, "Three-point boundary value problems for conformable fractional differential equations," J. Funct. Spaces, vol. 2015, p. 6, 2015, id/No 706383, doi: 10.1155/2015/706383. 
[6] M. Bouaouid, M. Hannabou, and K. Hilal, "Nonlocal conformable-fractional differential equations with a measure of noncompactness in Banach spaces," Journal of Mathematics, vol. 2020, 2020, doi: 10.1155/2020/5615080.

[7] M. Bouaouid, K. Hilal, and S. Melliani, "Sequential evolution conformable differential equations of second order with nonlocal condition," Advances in Difference Equations, vol. 2019, no. 1, pp. 1-13, 2019, doi: 10.1186/s13662-019-1954-2.

[8] M. Caputo and M. Fabrizio, "A new definition of fractional derivative without singular kernel," Progr. Fract. Differ. Appl, vol. 1, no. 2, pp. 1-13, 2015, doi: 10.12785/pfda/010201.

[9] A. Debbouche and D. Baleanu, "Controllability of fractional evolution nonlocal impulsive quasilinear delay integro-differential systems," Computers \& Mathematics with Applications, vol. 62, no. 3, pp. 1442-1450, 2011, doi: 10.1016/j.camwa.2011.03.075.

[10] A. Debbouche and D. Baleanu, "Exact null controllability for fractional nonlocal integrodifferential equations via implicit evolution system," Journal of Applied Mathematics, vol. 2012, 2012, doi: 10.1155/2012/931975.

[11] D. Ding, X. Zhang, J. Cao, N. Wang, and D. Liang, "Bifurcation control of complex networks model via PD controller," Neurocomputing, vol. 175, pp. 1-9, 2016, doi: 10.1016/j.neucom.2015.09.094.

[12] D. H. Hyers, "On the stability of the linear functional equation," Proceedings of the National Academy of Sciences of the United States of America, vol. 27, no. 4, p. 222, 1941, doi: 10.1073/pnas.27.4.222.

[13] F. Jarad, T. Abdeljawad, and J. Alzabut, "Generalized fractional derivatives generated by a class of local proportional derivatives," The European Physical Journal Special Topics, vol. 226, no. 16-18, pp. 3457-3471, 2017, doi: 10.1140/epjst/e2018-00021-7.

[14] R. Khalil, M. Al Horani, A. Yousef, and M. Sababheh, "A new definition of fractional derivative," Journal of Computational and Applied Mathematics, vol. 264, pp. 65-70, 2014, doi: 10.1016/j.cam.2014.01.002.

[15] A. A. Kilbas, H. M. Srivastava, and J. J. Trujillo, Theory and applications of fractional differential equations, 2006, vol. 204.

[16] V. Lakshmikantham, S. Leela, and J. V. Devi, "Theory of fractional dynamic systems." CSP, 2009.

[17] S. Meng and Y. Cui, "The extremal solution to conformable fractional differential equations involving integral boundary condition,” Mathematics, vol. 7, no. 2, p. 186, 2019, doi: 10.3390/math7020186.

[18] I. Podlubny, Fractional differential equations: an introduction to fractional derivatives, fractional differential equations, to methods of their solution and some of their applications. Elsevier, 1998.

[19] M. A. Ragusa and A. Razani, "Weak solutions for a system of quasilinear elliptic equations," arXiv preprint arXiv:2006.05262, 2020.

[20] M. A. Ragusa and A. Scapellato, "Mixed Morrey spaces and their applications to partial differential equations," Nonlinear Analysis: Theory, Methods \& Applications, vol. 151, pp. 51-65, 2017, doi: 10.1016/j.na.2016.11.017.

[21] M. A. Ragusa and A. Tachikawa, "Regularity for minimizers for functionals of double phase with variable exponents," Advances in Nonlinear Analysis, vol. 9, no. 1, pp. 710-728, 2019, doi: 10.1515/anona-2020-0022.

[22] K. M.-B. Ross, "An introduction to the fractional calculus and differential equations," A Wiley Interscience Publication, 1993.

[23] S. G. Samko, A. A. Kilbas, O. I. Marichev et al., Fractional integrals and derivatives. Gordon and Breach Science Publishers, Yverdon Yverdon-les-Bains, Switzerland, 1993, vol. 1.

[24] D. Smart, "Fixed point theorems, Cambridge university," Press, Cambridge, 1980. 
[25] J. Wang and H. M. Ahmed, "Null controllability of nonlocal Hilfer fractional stochastic differential equations," Miskolc Mathematical Notes, vol. 18, no. 2, pp. 1073-1083, 2017, doi: 10.18514/MMN.2017.2396.

[26] W. Zhong and L. Wang, "Basic theory of initial value problems of conformable fractional differential equations," Advances in Difference Equations, vol. 2018, no. 1, pp. 1-14, 2018, doi: 10.1186/s13662-018-1778-5.

\section{Author's address}

\section{Mohamed I. Abbas}

Alexandria University, Faculty of Science, Department of Mathematics and Computer Science, Alexandria, 21511, Egypt

E-mail address: miabbas@alexu.edu.eg; miabbas77@gmail.com 